\title{
Linx
}

Revue des linguistes de l'université Paris X Nanterre

40 | 1999

Le statut d'unité lexicale

\section{Andrée Borillo, L'espace et son expression en français}

Ophrys, 1998

Jean-François Jeandillou

\section{CpenEdition}

Journals

Édition électronique

URL : http://journals.openedition.org/linx/802

DOI : 10.4000/linx.802

ISSN : 2118-9692

Éditeur

Presses universitaires de Paris Nanterre

Édition imprimée

Date de publication : 1 juin 1999

Pagination : 263-265

ISSN : 0246-8743

Référence électronique

Jean-François Jeandillou, « Andrée Borillo, L'espace et son expression en français », Linx [En ligne], 40 | 1999, mis en ligne le 22 juin 2012, consulté le 22 septembre 2020. URL : http:// journals.openedition.org/linx/802; DOI : https://doi.org/10.4000/linx.802

Ce document a été généré automatiquement le 22 septembre 2020

Département de Sciences du langage, Université Paris Ouest 


\section{Andrée Borillo, L'espace et son expression en français}

Ophrys, 1998

Jean-François Jeandillou

\section{RÉFÉRENCE}

Andrée Borillo : L'espace et son expression en français, Ophrys, 1998

$1 \quad$ Il est des sujets privilégiés en linguistique. L'espace est de ceux-là. Sans doute parce que la perception est sollicitée, que le sujet parlant, point de référence, est souvent au coeur du problème, que le paramètre temporel intervient, et surtout, que les concepts utilisés sont clairs. De ce fait, la lecture n'est nullement gênée par le caractère technique de quelques règles transformationnelles.

2 Car il s'agit bien d'une étude rigoureuse et non d'une simple description agréable de notre expérience sensible où les relations spatiales sollicitent des lieux, des êtres ou des objets. Différentes classes grammaticales sont en jeu comme les prépositions et les verbes, les premières ayant un rôle prépondérant dans les relations statiques entre lieux et êtres ou objets, tandis que les verbes sont la clef de voûte des relations dynamiques ; des structures syntaxiques de phrases locatives type se dessinent souvent, dépendantes de facteurs aspectuels, telle la nature du procès ; une propriété comme la mobilité du complément prépositionnel semble corrélée à la présence de traits dans le verbe (indication sur la position, manière d'être, etc.). La prédominance des propriétés binaires contribue à donner une représentation «transparente » de l'espace : site vs cible, statique vs dynamique, localisation interne vs externe, préposition de localisation vs préposition dynamique, orientation situationnelle vs canonique, polarité positive vs négative. Avec les verbes, le schéma devient ternaire, puisque le déplacement peut connaître une phase initiale, une phase médiane et une phase finale. 
Cependant, le tableau se complique, notamment au moment de la combinatoire entre certaines unités. Car si certaines propriétés des prépositions sont intrinsèques, d'autres varient au gré des verbes. Les propriétés elles-mêmes des différents acteurs soulèvent certaines difficultés. Ainsi, la frontière entre lieu et objet ne se dessine pas toujours avec netteté, en particulier dans le cas des portions d'espace. La granularité variable de certains objets s'oppose aux propriétés géométriques bien établies d'autres : une rivière sera tantôt considérée comme un volume, tantôt comme une surface, tantôt encore comme une ligne. Les propriétés des noms et des adjectifs sont fortement dépendantes de la perception du sujet : le devant d'un objet sans orientation propre n'acquiert l'appellation de « devant » qu'en fonction de la place du locuteur par rapport à celui-ci. Enfin, des considérations contextuelles influent sur l'interprétation de l'orientation de certains objets, tels les rochers.

4 Et pourtant, malgré la complexité des variables, dans un souci d'humilité l'auteur insiste en introduction et en conclusion sur le caractère général de la description et sur la prise en compte de faits déjà connus. Certes, des notions comme celles de verbes statiques/dynamiques, de rôle du sujet parlant font désormais figure de classique dans ce domaine. Mais des notions plus modernes sont exploitées avec originalité, telle la polarité ou encore la règle transformationnelle de restructuration. Cette dernière apparaît sous un jour nouveau (p. 76) : dans cette relation partie-tout, le substantif fond correspondant à la partie (Il y a des sièges dans le fond de la pièce) se trouve rejeté à l'extérieur du groupe nominal $\mathrm{N}$ de $\mathrm{N}$, après une virgule (Il y a des sièges dans la pièce, au fond). La notion de congruence entre verbe et préposition est introduite : elle caractérise les cas où les types de phase (initiale, médiane, finale) décrits par la préposition et le verbe convergent. Sans être systématiquement définies, ces notions sont illustrées avec une limpidité qui pallie l'absence de glossaire.

On regrettera qu'aucune mention ne soit faite des travaux de Salkoff (p.148) à propos des constructions dites «standard" et «croisée» en anglais, alors qu'une étude ultérieure de Levin est citée pour la même langue. Boons, Guillet, Leclère 1976 sont bien cités dans le corps du texte, mais n'apparaissent pas dans la bibliographie. Enfin, mentionnons quelques coquilles çà et là : un elle pour il dans l'introduction (IV), un $s$ manquant à difficile (p. 128), il quitté au lieu de il a quitté (p. 136), la déterminant chat (p. 137), struture au lieu de structure (p. 141), s'interprèter (p. 141), des relation au singulier (p. 166), pour citer l'essentiel. Ces coquilles font figure de détail, tout comme la confusion qui entoure à côté de, classé tantôt comme préposition, tantôt comme adverbe.

En effet, cela ne saurait altérer l'image que donne ce livre : en dépit de la complexité du problème, l'expression de l'espace en français devient limpide. Car Andrée Borillo a su allier l'intuition d'études comme celles de Vandeloise et la rigueur des analyses de Guillet et Leclère. C'est là que réside son originalité. 


\section{AUTEURS}

JEAN-FRANÇOIS JEANDILLOU

Université de Paris X Nanterre 